\title{
Investigation of the Sudden Solar Ionospheric Disturbance using Radio Telescope
}

\author{
Adam Aqasha ${ }^{1}$, Andrien Zheng ${ }^{1}$, Sneha Athreya ${ }^{2 *}$, and Hoe Teck Tan ${ }^{1}$ \\ ${ }^{1}$ School of Science and Technology, 1 Technology Drive, Singapore \\ ${ }^{2}$ National Junior College, 37 Hillcrest Road, Singapore
}

\begin{abstract}
Low-frequency radio telescopes are cheap and useful devices for the investigation of terrestrial and extra-terrestrial emissions. These emissions come either from the Sun and the planet Jupiter to terrestrial emissions. This project aims to investigate the Very Low Frequency (VLF) waves from mid-August to October 2019 using Radio JOVE (20 MHz) and SSID (3-30 kHz) to observe for the occurrence of solar flares and see how if the radio telescopes that the team set up is reliable. This will allow us future students aspiring to learn about astronomy to examine solar flares in detail during the upcoming solar maximum. Not many flares were detected as this period happens to be a solar minimum. However, a series of flares occurred between 30 September 2019 and 1 October 2019, which the telescopes have been able to detect, particularly SSID.
\end{abstract}

\section{Introduction}

Solar events have great influences over the Solar System. On Earth, the understanding of these events is paramount. They produce streams of high energy particles which greatly affect the Earth's space weather and may cause hazards to the space-stations, the spacecrafts and the astronauts. In addition, massive solar events may also affect the terrestrial systems. They sometimes post dangers to the satellite systems as well as causing blackouts to the electric power grids.

In this paper, we focus on the solar activities like the solar flares and the coronal mass ejections. These activities cause disturbances to the ionosphere. The ionosphere is responsible for the reflection of radio waves. Thus, any disturbance to the ionosphere will affect the reflection of the radio waves. We investigate how these solar events affect the radio waves in the Very Low Frequency regime.

\subsection{The Ionosphere}

The ionosphere is an ionized layer in the atmosphere that is around 50 to $600 \mathrm{~km}$ above the Earth's surface. It is caused by the UV radiation that comes from the Sun. The degree of ionization changes due to many factors, such as latitude, height above ground or time of the day. During the day, UV radiation that is received from the sun is high, causing a greater degree of ionization, while at night, the electrons and ions recombine and the level of ionization decreases. There is also a greater degree of ionization closer to the equator as these

\footnotetext{
*Email: snehaathreya03@gmail.com
} 
regions receive a higher and more concentrated amount of UV radiation from the Sun. The ionosphere consists of 3 main layers-D, E and F. The following table describes the properties of these layers:

Table 1: Layers of the Ionosphere

\begin{tabular}{|l|l|l|l|l|}
\hline Layer & Altitude & $\begin{array}{l}\text { Time it is } \\
\text { present }\end{array}$ & $\begin{array}{l}\text { Primary ionizing } \\
\text { radiation }\end{array}$ & Effect on VLF \\
\hline D & $60-90 \mathrm{~km}$ & Day & $\begin{array}{l}\text { Hard X-rays } \\
(0.1-1 \mathrm{~nm})\end{array}$ & $\begin{array}{l}\text { Absorption/ } \\
\text { Reflection }\end{array}$ \\
\hline E & $100-125 \mathrm{~km}$ & Day and night & $\begin{array}{l}\text { Soft X-rays } \\
(1-20 \mathrm{~nm}), \text { or } \\
\text { Extreme UV } \\
(80-103 \mathrm{~nm})\end{array}$ & Reflection \\
\hline F & $300-400 \mathrm{~km}$ & Day and night & $\begin{array}{l}\text { Extreme UV } \\
(20-80 \mathrm{~nm})\end{array}$ \\
\hline
\end{tabular}

The ionosphere is a great propagator of radio waves. Monitoring the power of these radio signals allows us to detect changes in the ionosphere. When a solar storm occurs, the D region of the ionosphere drastically reaches a high level of ionization, allowing greater reflection of the Very Low Frequency (VLF) waves. This results in the radio signals showing a sudden, sharp spike upwards. We monitor VLF as it is readily available all over the world and because it is transmitted at constant power. $[1,5,6]$

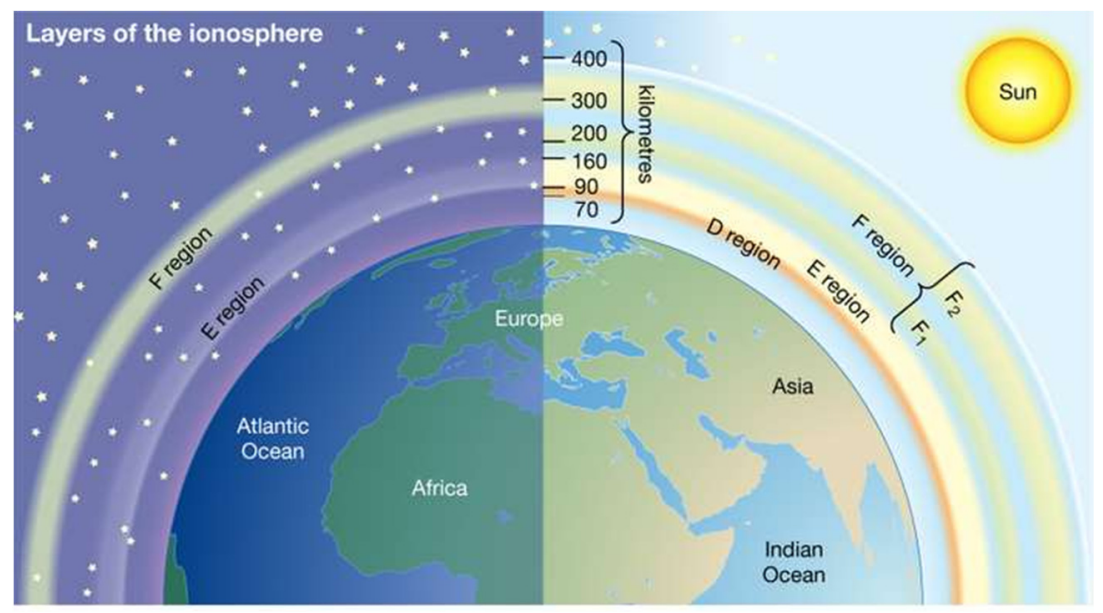

Figure 1: Layers of the Ionosphere [11] 


\subsection{Solar Flares}

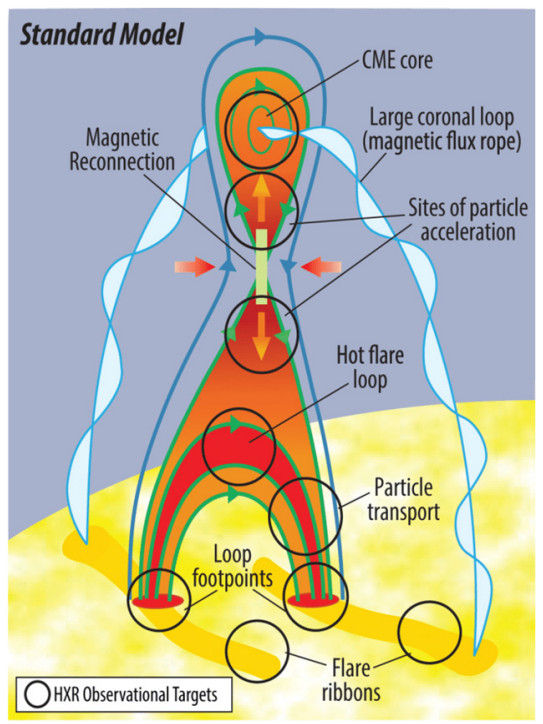

Figure 2: Model of Solar Flare [12]

Solar flares are massive explosions on the Sun that occur when charged particles in the Sun's plasma erupt into space, travelling with enormous speed. These flares occur when the magnetic field lines of the sun reconnect, as seen in Figure 2, releasing large amounts of energy and X-ray waves into space. It usually occurs in the active regions of the sun, near sunspots, along the dividing line between areas of oppositely directed magnetic fields, where temperatures might even reach more than 10,000 Kelvin. Depending on the location and trajectory of the solar flares, the X-rays that are shot out may create electrical disturbances in the ionosphere of the Earth. The process of the X-rays colliding with earth and disturbing its ionized layer is known as solar storms.

One of the huge dangers of a solar flare is widespread electrical disruption. When the particles strike the Earth's magnetosphere, they could produce an electrical charge, an electrical charge that is powerful enough to travel to the planet's surface. When those charged currents encounter electrical grids, it may lead to complications such as power faults which may affect cities or even whole countries. On 12 March 1989, a particularly strong solar flare struck North America and overwhelmed the electrical grid of the Canadian province of Quebec. At 2:44 a.m. the following morning, a series of cascade failures occurred in the electrical system, resulting in a province-wide blackout that lasted 12 hours.

We believe that a future solar storm might be even more devastating as there are a lot greater advancements in technologies currently as compared to 1989. This makes us more vulnerable as more systems depend on electricity to function properly. Due to the immense impact these solar storms can have on business, telecommunication systems, and other aspects of our life, we found that ionospheric disturbances are an interesting study and hence the focus of our project $[2,5,6]$. 
The different categories of solar flares are:

Table 2: Classes of Solar Flares

\begin{tabular}{|l|l|lll|}
\hline $\begin{array}{l}\text { Solar } \\
\text { Flare }\end{array}$ & X-ray flux (Log Watts/ Sq. m) & Effect & & \\
\hline X & -4 & $\begin{array}{l}\text { Causes very serious damage to radio } \\
\text { communications, even blackouts. }\end{array}$ \\
\hline M & -5 & $\begin{array}{l}\text { Causes some impact on radio } \\
\text { communications. }\end{array}$ \\
\hline C & -6 & Minor & & \\
\hline B & -7 & None & & \\
\hline A & -8 & None & & \\
\hline
\end{tabular}

\subsection{Coronal Mass Ejections (CME)}

During Coronal Mass Ejections (CMEs), enormous bubbles of superheated gas - called plasma - are ejected from the Sun. Over several hours, they can eject billions of tons of coronal material off the Sun's surface and accelerated to speeds of a million miles per hour (1.6 million kilometres per hour) and carry an embedded magnetic field (frozen in flux) that is stronger than the background solar wind interplanetary magnetic field (IMF) strength. The fastest Earth-directed CMEs can travel to our planet in as short as 15-18 hours. Slower CMEs might take several days to arrive. They expand in size as they propagate away from the Sun and larger CMEs can reach a size comprising nearly one-quarter of the space between Earth and the Sun by the time it reaches our planet. CMEs can happen several times a day when the Sun is most active, whereas, during its fewer active periods, CMEs could occur only about once every five days $[3,6]$.

\section{Aim}

Our aim for the project is to test if the telescopes installed accurately receive signals and can detect the occurrence of solar flares.

\section{Methodology}

A Radio JOVE $(20 \mathrm{MHz})$ and SSID $(3-30 \mathrm{kHz})$ receiver was connected to the computer directly to continuously monitor the ionosphere. Radio JOVE was set up (figure 1) at National Junior College (NJC) while the SSID was placed at the School of Science and Technology (SST). The signal strength was monitored for any disturbances and counter checked with data from earth and space weather forecasts. The archived data would then be used to confirm our results about the disturbances. 


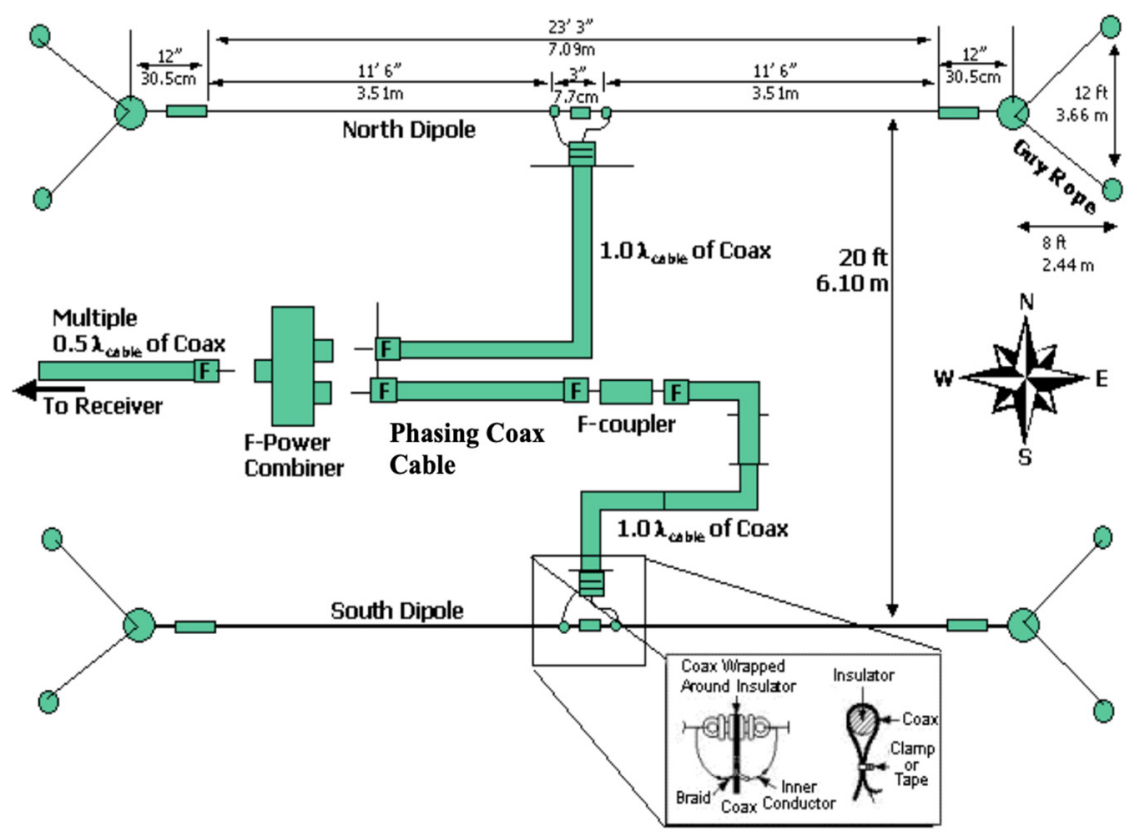

Figure 1: Setup of Radiojove

(source: https://radiojove.gsfc.nasa.gov/telescope/ant manual.pdf) [4]

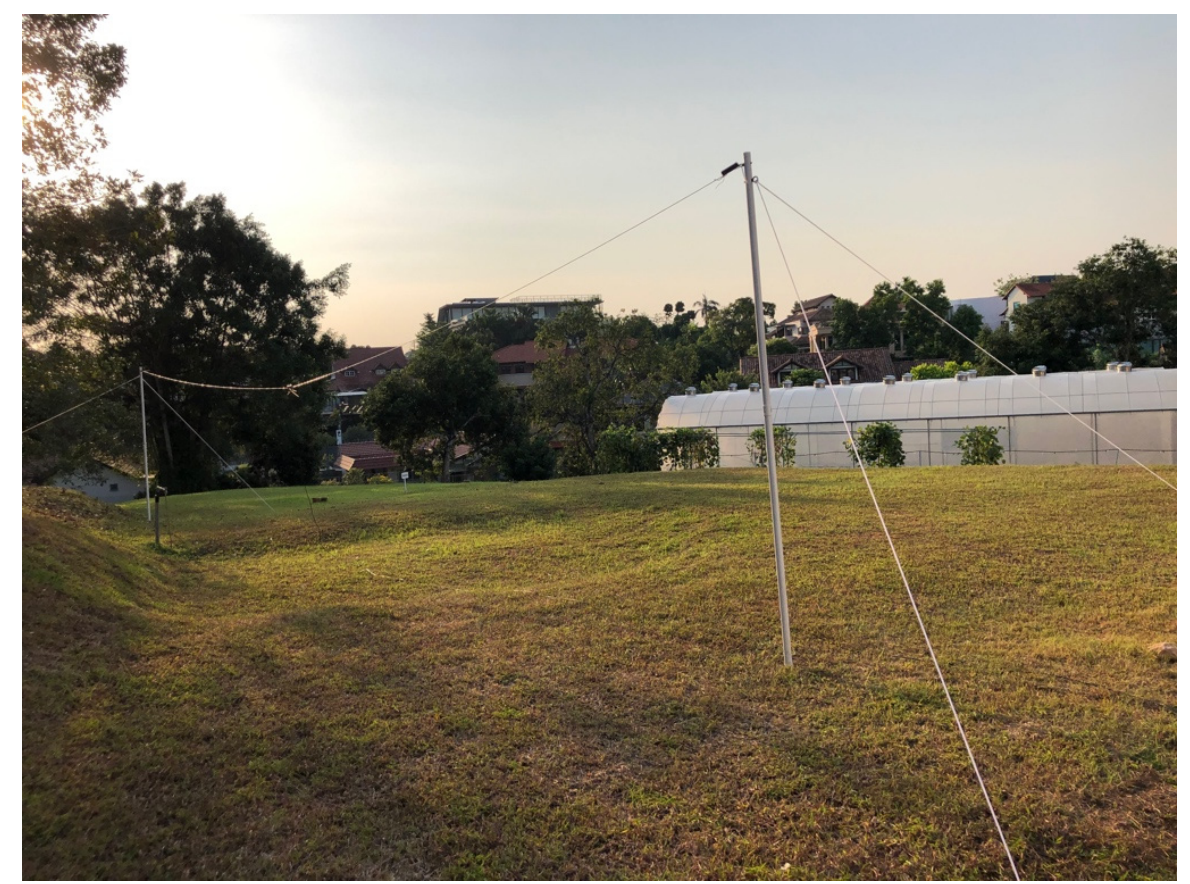

Figure 2: Deployment of Radiojove 

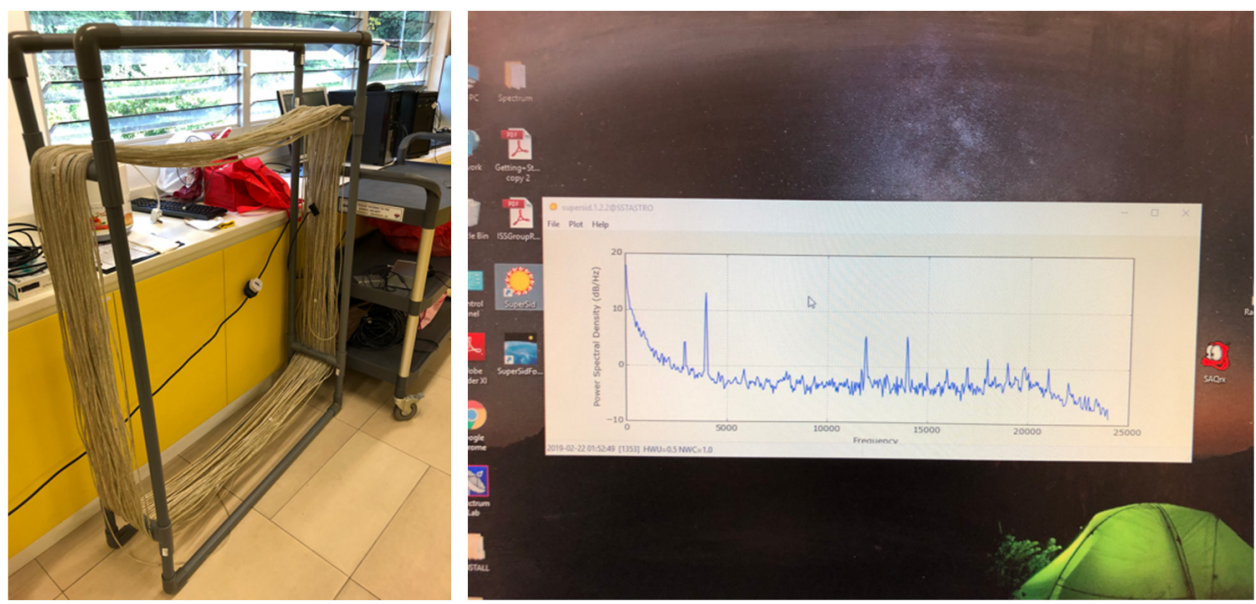

Figure 3: Deployment of the SSID

\section{Results and Discussion}

The following are the graphical images obtained using the Skypipe 2 software for Radio JOVE and software for SSID. Since the period of data collection happens to be during a solar minimum, there was a lack of flares observed. However, a small series of flares may have been detected by the telescopes from 30 September 2019 to 1 October 2019, of which the results shall be shown.

The graphs for Radio JOVE show changes in radio signal against local time in Singapore, while SSID shows the change in radio signals against Coordinated Universal Time (UTC), which is 8 hours behind Singapore. 
Table 3: Graphs showing strength of radio signals recorded by Radio JOVE and SSID

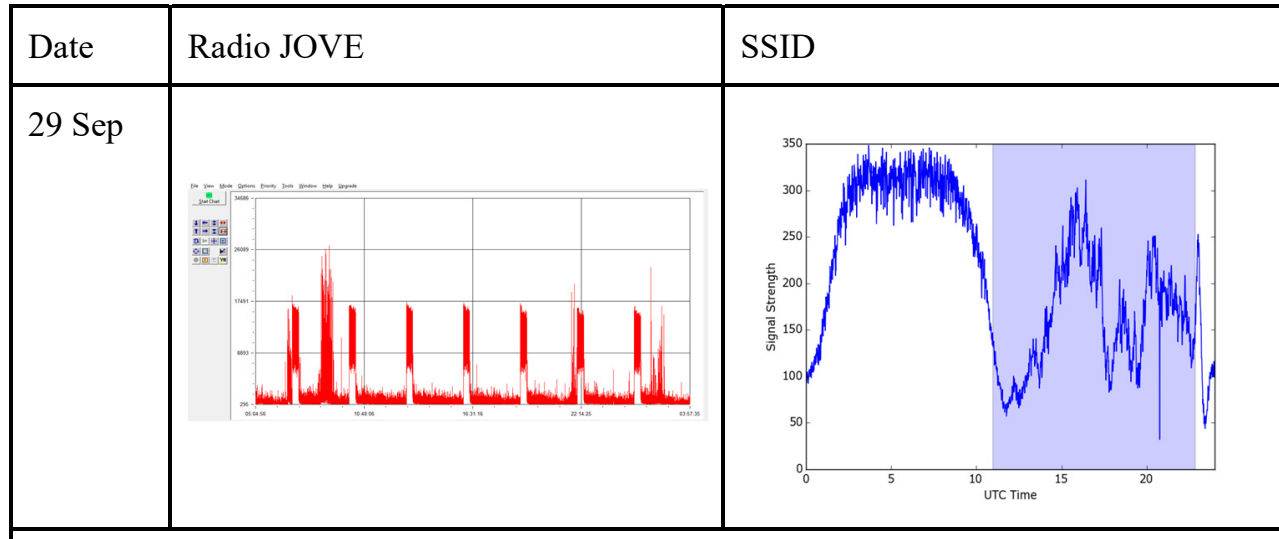

Maximum flux: A8.64

No flares reported by https:/www.spaceweatherlive.com/

Background flux: A4.7 [7]

Low signal strength recorded by SSID of no more than 350 . Some disturbance recorded by Radio JOVE around 8am (0:00 UTC on 29 September).
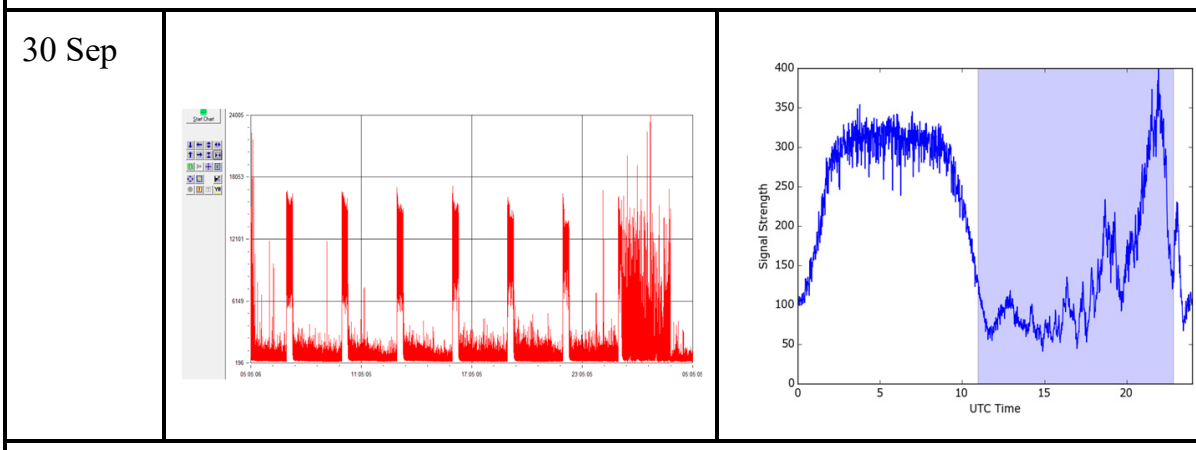

Maximum flux: B1.57

No flares reported by https://www.spaceweatherlive.com/

Background flux: A2.6 [8]

The SSID graph shows a high peak (Signal strength: 400) after 20:00 UTC during the occurrence of the solar flares. 20:00 UTC corresponds to 4am in Singapore on 1 of October, which is 8 hours ahead.

The graph on the left shows results from $5 \mathrm{am}$ on 30 September to 5 am on 1 October. There was a high amount of disturbance from around $2 \mathrm{am}$ to $4 \mathrm{am}$ that could be attributed to the occurrence of a solar flare. 


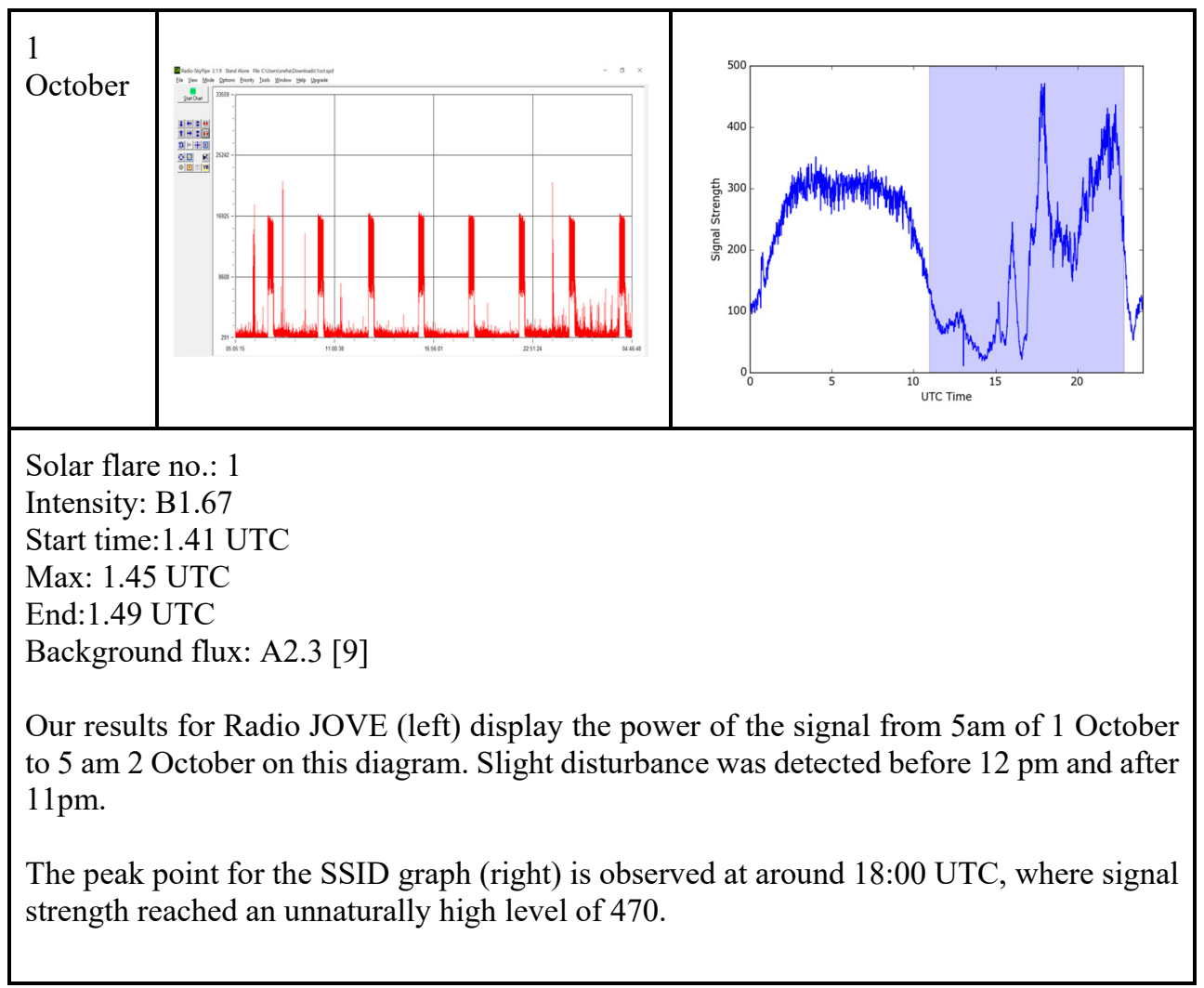

Another source that had confirmed the occurrence of the solar flares at this time was ISRO's (Indian Space Research Organization) website, a more reliable source than https://www.spaceweatherlive.com/ [10].

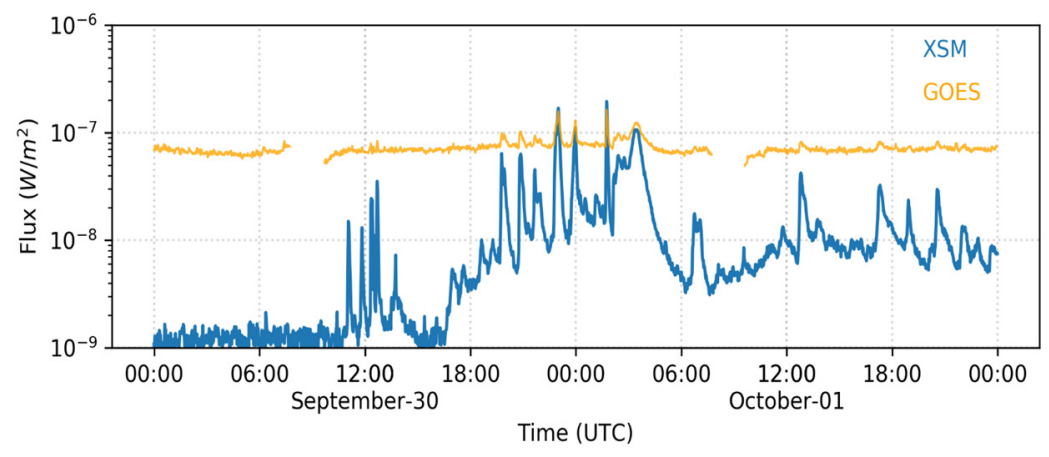

Figure 4: Graph showing the solar X-ray flux as measured by XSM (in blue) and flux measured by X-ray sensor on Geostationary Operational Environmental Satellite (GOES15) (in orange) 
Figure 4 shows that there indeed was a series of flares detected from 30th September 2019 00:00 UTC - 1st October 2019 23:59 UTC by the Solar X-ray Monitor (XSM) that was carried by Chandrayaan-2 orbiter. Space Weather Live had only identified the occurrence of 1 solar flare during this period; this may have been because the receiver was facing away from the sun while the flare occurred. Singapore was also facing away from the sun when the solar flares began on 29 September (around 12:00 UTC) but the telescopes managed to observe some spikes on 1 October during the daytime. This shows that the telescopes were successful in detecting the flares.

\section{Conclusion}

Solar flares of B-class were detected by our telescopes. The regular disturbances for the Radio JOVE make it difficult for us to pinpoint the time from which the solar flares may have begun. Due to the disturbances and the uncertainty for what caused them, our results for Radio JOVE may be not as reliable as our results from SSID.

\section{References}

[1] Tracking Solar Flares. (n.d.). Retrieved 2019, from http://solarcenter.stanford.edu/SID/activities/ionosphere.html

[2] Kazmeyer, M. (2019, March 2). What Effects Can Solar Flares Have Directly on the Earth? Retrieved 2019, from https://sciencing.com/effects-can-solar-flares-directly-earth3864.html

[3] What is a coronal mass ejection? (n.d.). Retrieved July 2019, from https://earthsky.org/space/what-are-coronal-mass-ejections

[4] National Aeronautics and Space Administration. (2012). Radio Jove RJ1.2 Antenna Kit Assembly Manual. Retrieved

https://radiojove.gsfc.nasa.gov/telescope/ant_manual.pdf

[5] Arnold, S. (2014). Getting Started in Radio Astronomy. The Patrick Moore Practical Astronomy Series. doi: 10.1007/978-1-4614-8157-7

[6] Andrews, P. (2011). How to Build Your Own Radio Telescope (3rd ed.).

[7] Solar flares - Sunday, 29 September 2019. (n.d.). Retrieved 2019, from https://www.spaceweatherlive.com/en/archive/2019/09/29/xray

[8] Solar flares - Monday, 30 September 2019. (n.d.). Retrieved 2019, from https://www.spaceweatherlive.com/en/archive/2019/09/30/xray

[9] Solar flares - Tuesday, 1 October 2019. (n.d.). Retrieved 2019, from https://www.spaceweatherlive.com/en/archive/2019/10/01/xray

[10] Solar flare observed by the Solar X-ray Monitor on Chandrayaan-2. (n.d.). Retrieved December 2019, from https://www.isro.gov.in/update/10-oct-2019/solar-flare-observedsolar-X-ray-monitor-chandrayaan-2

\subsection{Images}

[11] $\quad$ https://cdn.britannica.com/s:700x500/46/109746-050-9511BBEF/differenceslayers-ionosphere-Earth.jpg [12] https://www.researchgate.net/figure/HXR-observations-emission-from-allkeypoints-in-the-standard-model-of-solar-eruptive fig1 312061210 